\title{
ACKNOWLEDGMENT TO OUR REFEREES
}

(C) Marta Olivetti Belardinelli and Springer-Verlag 2010

We would like to thank all those researchers that have served as referees for Cognitive Processing in 2008 and 2009:

Kais Al Naimee
Igor Aleksander
Tracy Alloway
Jean Marie Annoni
Alessandro Antonietti
Tito Arecchi
Alarik Arenander
Marios Avraamides
Christian Balkenius
Guido Band
Lawrence Barsalou
Andreas Bartels
Canan Basar-Eroglu
Demis Basso
Christian Werner
$\quad$ Becker-Asano
Jens F. Beckmann
Anna Belardinelli
Jason Bell
Guglielmo Bellelli
Sieghard Beller
Eshel Ben- Jacob
Jose Luis Bermudez
Emmanuelle Boloix
Michael H Brill
Vicki Bruce
Monica Bucciarelli
B. Rael Cahn
Maria Casagrande
Nicholas Cassimatis
Monica Castelhano
Paolo Cherubini
Ana B. Chica
Marzena Ciszak
Neal J. Cohen
Michael X Cohen
Elias Cohen
Michael Corballis
Alessandro
Moumer
Aian

Kais Al Naimee

Tracy Alloway

Jean Marie Annoni

Alessandro Antonietti

Tito Arecchi

Alarik Arenander

Marios Avraamides

Christian Balkenius

Guido Band

Lawrence Barsalou

Andreas Bartels

Canan Basar-Eroglu

Demis Basso

Christian Werner

Becker-Asano

Jens F. Beckmann

Anna Belardinelli

Jason Bell

Guglielmo Bellelli

Sieghard Beller

Eshel Ben- Jacob

Emmanuelle Boloix

Michael H Brill

Monica Bucciarelli

B. Rael Cahn

Casagrande

Nicholas Cassimatis

Monica Castelhano

Paolo Cherubini

Ana B. Chica

Marzena Ciszak

Neal J. Cohen

Michael X Cohen

Elias Cohen

Michael Corballis

essandro

Simone Cutini
Alessandro D'Ausilio

Clare Davies

Luigi De Gennaro

Michel Denis

Angelo Di Garbo

Rosalia Di Matteo

Alex Doumas

Matthew J. Dry

Annapaola Ercolani

Edgar Erdfelder

K. Anders Ericsson

Jonathan St B T Evans

Alessandro Farini

Stefano Federici

Alexander Fingelkurts

Luigi Fortuna

Mattia Frasca

Walter J. Freeman

Christian Freksa

Vinciane Gaillard

Nicholas A. Giudice

Joseph Glicksohn

Noah Goodman

Thomas Hünefeldt

Peter Hancock

John R. Hayes

Ulrich Hoffrage

Autumn B. Hostetter

Jörn Hounsgaard

Saskia Jaarsveld

Petr Janata

Niels Janssen

Viktor Jirsa

Philip Johnson-Laird

J. Scott Jordan

Scott Kelso

Wlodzimierz Klonowski

Pia Knoeferle

Ljupco Kocarev

Stefan Kopp

Ulrich Krohs

Edmundo Kronmuller
Juergen Kurths

Thomas Lachmann

Giulio Lancioni

Anton E. Lawson

Paul Lewis

Oliver Lindemann

Giuseppe Liotta

Robert H Logie

Alexander Logvinenko

Tania Lombrozo

Alessandro Londei

Fabio Lucidi

Juan Lupianez

Riccardo Manzotti

Jacques Marescaux

Jessecae Marsh

Francesco S. Marucci

E.J. Masicampo

Giuliana Mazzoni

Ryan Thomas McKay

Ken McRae

Patrizia Mecocci

Riccardo Meucci

Stephen Mitroff

Paolo Moderato

Carmela Morabito

Alain Morin

Daniele Nardi

Davide Nardo

Andrey Nikolaev

Matthijs Leendert Noordzij

Marta Olivetti

Belardinelli

Tom Ormerod

Massimiliano Palmiero

Maria Teresa Pazienza

Francesca Pazzaglia

Catherine Pelachaud

Jean-Luc Petit

Olimpia Pino

Jordan Pop-Jordanov
Antonino Raffone

Keith Rayner

Ronald Rensink

Antti Revonsuo

Thomas Reydon

Massimo Riani

Helge Ritter

Miguel Sanjuan

Walter Sannita

Valerio Santangelo

Julio Santiago

Marcia Scherer

Ute Schmid

Caroline Schnakers

Carlo Semenza

Itay Shani

Lawrence Shapiro

Dikla Shmueli

Alcino J. Silva

Hannah Smithson

Michael J Spivey

Narayanan Srinivasan

Boris Suchan

Holly A. Taylor

Thomas Toppino

Fred Travis

Jeroen J.A. van Boxtel

Maaike van den Haak

Cees van Leeuwen

Erik van Loosbroek

Iris van Rooij

Ricardo Viana

David Vinson

Kai Vogeley

Momme von Sydow

Geoff Ward

Gezinus Wolters

Rene Zeelenberg

Hubert D. Zimmer 